\title{
Modelo de simulación para procedimientos guiados por ultrasonografía: bloqueos neuroaxiales de la región lumbar
}

\author{
Simulation model for ultrasonography-guided procedures: \\ neuraxial blocks of the lumbar region \\ Santiago Maffia Bizzozero, ${ }^{*}$ Nicolás Ezequiel Banti, ${ }^{*}$ Eduardo Teragni*
}

\footnotetext{
Palabras clave:

Simulador, bloqueos, región lumbar, entrenamiento médico.

Key words: Simulator, blocks, lumbar region, medical training.
}

* Centro Universitario de Biosimulación Clínica;

Laboratorio de Recursos Instruccionales;

Primera Cátedra del Departamento de Anatomía. Facultad de Medicina de la Universidad de Buenos Aires.

Recibido: 13/02/2019 Aceptado: 15/03/2019

\section{RESUMEN}

El concepto de simulación refiere a la representación de algo, imitando lo que no es; simular en el área de las ciencias médicas será situar un estudiante en un contexto que imite algún aspecto de la realidad clínica. Dado que la columna vertebral se encuentra localizada en la profundidad, la obtención de imágenes por ultrasonografía obtenidas mediante ultrasonido de baja frecuencia, resulta muy útil a la hora de realizar procedimientos invasivos guiados por esta técnica. Las habilidades necesarias para realizar el procedimiento de punción o bloqueo neuroaxial central guiado por ultrasonido requiere de un entrenamiento y práctica intensivos. Por ello el trabajo tuvo como meta el diseño y la construcción de un simulador para la enseñanza de bloqueos neuroaxiales de la región lumbar guiados por ultrasonografía. Se desarrolló un simulador para el entrenamiento en bloqueos neuroaxiales guiados por ultrasonografía. Este modelo brinda una imagen de características muy similares a las obtenidas mediante el uso de una sonda lineal sobre pacientes reales, además de las características de los distintos tejidos cuando se practican punciones en la región lumbar. Este simulador es uno de los primeros disponibles con estas características y construido con materiales nacionales a un costo sensiblemente menor al de los disponibles actualmente en el mercado, inscribiéndose dentro de los disponibles para el entrenamiento médico basado en técnicas de simulación. Las mismas tienen como objeto que el profesional de la salud adquiera habilidades que le sean de utilidad en su práctica clínica. En la actualidad el principal desafío que enfrentamos en el campo de la educación médica es generar investigaciones, diseños y desarrollos de calidad tendiente a generar una forma más efectiva y eficiente de usar la simulación en educación médica que optimice el uso de recursos y aporte a la formación profesional de calidad.

\section{ABSTRACT}

The concept of simulation refers to the representation of something, imitating what it is not; to simulate in the area of medical sciences will be to place a student in a context that mimics some aspect of clinical reality. Since the spine is located in the depth, the obtaining of images by ultrasound obtained by low frequency ultrasound is very useful when performing invasive procedures guided by this technique. The skills needed to perform the procedure of puncture or central neuraxial lock guided by ultrasound requires intensive training and practice. Therefore, the goal of the work was the design and construction of a simulator for the teaching of neuroaxial blocks of the lumbar region guided by ultrasonography. A simulator was developed for training in neuroaxial blocks guided by ultrasonography. This model provides an image of characteristics very similar to those obtained through the use of a linear probe on real patients, in addition to the characteristics of the different tissues when punctures are made in the lumbar region. This simulator is one of the first available with these characteristics and built with national materials at a significantly lower cost than those currently available in the market, enrolling among those available for medical training based on simulation techniques. The purpose of the same is for the health professional to acquire skills that are useful in their clinical practice. Currently, the main challenge we face in the field of medical education is to generate research, designs and quality developments aimed at generating a more effective and efficient way to use simulation in medical education that optimizes the use of resources and contributes to the quality professional training.

\section{INTRODUCCIÓN}

El Informe Flexner (1910) generó una de las primeras innovaciones en la educación médica moderna e introdujo un cambio paradigmático en el modo de enseñar las ciencias médicas, lo que impulsó numerosas reformas en las escuelas de Medicina en Estados Unidos y Canadá. A partir de la segunda mitad del siglo $X X$, se introdujo en el campo de la educación médica el concepto de "entrenamiento basado en simulación", que ha representado, sin 
dudas, un salto cualitativo en dicho campo., ${ }^{1,2}$ El concepto "simulación" refiere la representación de algo, imitando lo que no es; "simular" en el área de las ciencias médicas será situar a un estudiante en un contexto que imite algún aspecto de la realidad clínica. Este concepto no es nuevo, ya que existen antecedentes al menos desde el siglo XVIII, cuando Angélique Marguerite du Coudray (1714-1794) recorrió Francia enseñando a las mujeres el oficio de partear; a ella se le atribuye la invención de uno de los primeros simuladores obstétricos para practicar partos. ${ }^{1}$ Desde entonces han aparecido numerosos modelos de simuladores para entrenarse en habilidades. En 1960, Asmund Laerdal diseñó un maniquí para enseñar destrezas durante un paro cardiorrespiratorio. En el mismo año, Stephen Abrahamson y J. Denson construyeron un modelo que imitaba los ruidos cardiacos y pulmonares. En 1980, David Gaba diseñó un maniquí que brindaba respuestas fisiológicas a los medicamentos aplicados en la anestesia (Comprehensive Anaesthesia Simulation Environment-CASE), al que le siguió el simulador GAS (Gainesville Anaesthesia Simulator).

Cuando se utilizan las técnicas de simulación, quien aprende no trabaja directamente con el objeto de estudio, sino que lo hace con una representación del mismo, lo que le permite generar un aprendizaje significativo de las habilidades que requerirá en su práctica profesional. ${ }^{2}$

Dado que la columna vertebral se encuentra localizada en la profundidad, la obtención de imágenes mediante ultrasonido de baja frecuencia resulta muy útil a la hora de realizar procedimientos invasivos guiados por esta técnica. Las habilidades necesarias para llevar a cabo la punción o bloqueo neuroaxial central guiado por ultrasonido requieren un entrenamiento y práctica intensivos por ser un área anatómicamente compleja y un procedimiento que no se encuentra exento de complicaciones. ${ }^{3,4}$

Habiendo identificado el problema al que se enfrenta el profesional que debe efectuarlo y teniendo en cuenta que el conocimiento de la anatomía de la región facilita su abordaje, se comenzó a pensar en el diseño de un fantoma (simulador) que combinara un alto grado de fidelidad anatómica con la reproducción de las formas y texturas de los tejidos de la región, que permitiera obtener imágenes ultrasonográficas similares a las obtenidas con pacientes reales y, también, que permitiera la repetición del procedimiento las veces que el operador requiriera hacerlo.

El presente trabajo tuvo como objetivo el diseño y construcción de un simulador para la enseñanza de bloqueos neuroaxiales de la región lumbar guiados por ultrasonografía, teniendo en cuenta la dificultad que plantea el aprendizaje de la técnica para efectuar dichos bloqueos y la adquisición de habilidades por parte de los profesionales para llevarlos a cabo; de este modo, la intervención tendió a generar un entorno de seguridad tanto para los estudiantes como para los pacientes.

El modelo tiene como antecedente el diseño y realización de un simulador para punciones lumbares "a ciegas" para su uso en centros de simulación y áreas de educación médica, el cual se rediseñó tomando como base las estructuras anatómicas desarrolladas y generando un nuevo diseño para que funcionara como un fantoma apto para efectuar entrenamientos en la ejecución de maniobras de punción guiadas por ultrasonografía.

\section{MATERIAL Y MÉTODOS}

Durante el año 2017 se llevó adelante el diseño y desarrollo del simulador. En primera instancia, se realizó un modelo de raquis lumbar en resina poliéster de alto impacto con agregado de carbonato de calcio a partir de un molde de caucho de siliconas tomado sobre estructuras óseas previamente ensambladas. Una vez que la pieza secó por completo, se procedió al desmolde de la misma; el tiempo promedio de secado fue de 12 a 24 horas. El agregado de carbonado de calcio a la resina permitió que resultara opaca a los rayos $X$ (radiopaca), por lo que el simulador fue apto para el examen radiológico de estructuras óseas. Las estructuras nerviosas se confeccionaron a partir de elementos tubulares de caucho de siliconas, y las partes blandas, el tejido celular subcutáneo y la piel, a partir de gel en aceite mineral cristalizado. Para ello se elaboró un molde de la superficie de la región lumbar tomado a partir de un modelo vivo; dicho molde fue confeccionado en alginato para impresiones. Para hacer la superficie cutánea en gel, se añadió a éste (en su fase líquida) colorante en polvo hasta lograr 
la coloración deseada. Luego de ello, se llevó a cabo el primer volcado sobre el molde. Las propiedades del gel hacen que este solidifique al descender su temperatura y equipararse con la del ambiente. Una vez lograda la solidificación del gel, se colocó la pieza de resina del raquis lumbar en posición anatómica y se ensamblaron las estructuras nerviosas sobre el mismo. Se tuvo especial cuidado en el posicionamiento de la totalidad de las estructuras, considerando sus relaciones anatómicas, su posición en el espacio y los espesores de la piel, el tejido celular subcutáneo y los planos musculares. Luego de realizado este procedimiento, se volcó el gel restante hasta completar la totalidad del molde. El tiempo promedio de solidificación del mismo fue de cuatro horas y 30 minutos. Luego de confeccionado el fantoma, se conservó a temperatura ambiente, alejado de la luz solar, durante 24 horas, con el objeto de que se estabilizaran sus componentes, y se montó el mismo sobre una base de MDF a la que se fijó mediante el empleo de silicona acética.

Sobre la base del desarrollo efectuado se lograron dos modelos básicos: uno tipo "almohadilla" para punción (Figura 1), de forma rectangular, y el otro, simulando la anatomía de superficie y topográfica (Figura 2). Ambos modelos brindan las mismas prestaciones en cuanto al modelo de simulación para el entrenamiento en bloqueos neuroaxiales del eje



Figura 1. Modelo desarrollado de un simulador para punción lumbar y bloqueos neuroaxiales bajo control ultrasonográfico: modelo anatómico. lumbar, como se ha descrito, con estructuras óseas palpables y radiopacas.

\section{RESULTADOS}

Se obtuvo por este método un simulador (fantoma) para la realización de bloqueos neuroaxiales guiados por ultrasonografía, que brinda una imagen de características muy similares a las logradas mediante el uso de una sonda lineal sobre pacientes reales (Figuras 3 y 4), además de las características de los distintos tejidos cuando se practican punciones en la región lumbar o la infiltración de las facetas articulares lumbares. Se trata de un modelo de uso específico y de baja tecnología (part-task trainers) diseñado para representar una parte del organismo y el ambiente, ${ }^{5}$ por lo que permite practicar habilidades básicas - en este caso, punciones lumbares o bloqueos neuroaxiales guiados por ultrasonografía.

El modelo diseñado, desarrollado y construido integralmente por los autores no se encuentra disponible en el país con las características mencionadas, sólo pueden encontrarse modelos importados de alto costo. El modelo desarrollado tiene un costo de producción de alrededor de un cuarto del precio al que se consiguen los

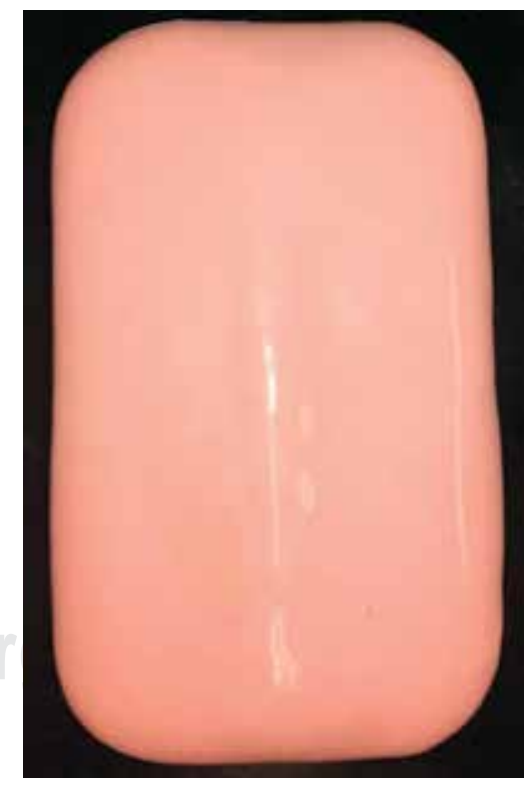

Figura 2. Modelo desarrollado de un simulador para punción lumbar y bloqueos neuroaxiales bajo control ultrasonográfico: modelo almohadilla para prácticas de procedimientos. 
modelos comerciales. El modelo fue probado en el curso teórico-práctico de Técnicas Intervencionistas para el Control del Dolor bajo Guía Ecográfica (Fundación Cenit, 2017); generó un excelente impacto entre los usuarios, tanto en los instructores (tutores) como en los cursantes. El modelo fue probado para realizar la localización ultrasonográfica de las estructuras del eje vertebral lumbar, y luego, para la punción mediante aguja del raquis para producir el bloqueo. Durante el entrenamiento, los cursantes podían llevar a cabo el rastreo ultrasonográfico en un modelo vivo para comparar la técnica simulada. En una encuesta hecha a 147 estudiantes que utilizaron la simulación para el entrenamiento procedimental en estas técnicas, $69(47 \%)$ respondieron que consideraban muy útil el uso del simulador; 53, útil (36\%); 17, escasamente útil, y ocho, inútil. En relación con la mejora en

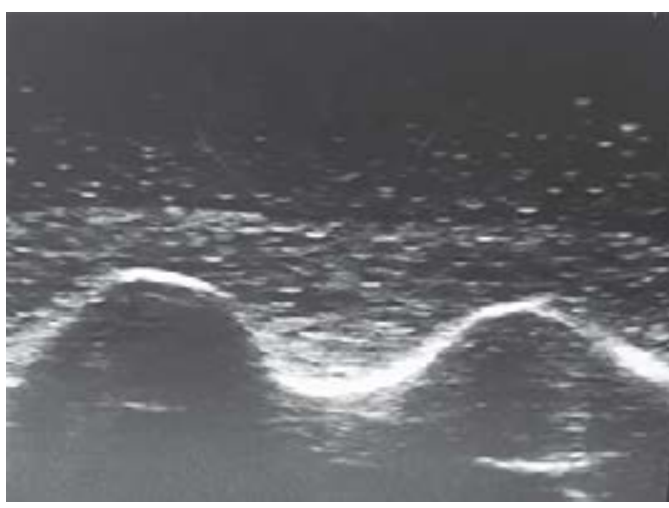

Figura 3. Imagen ultrasonográfica obtenida del simulador desarrollado: transversal.

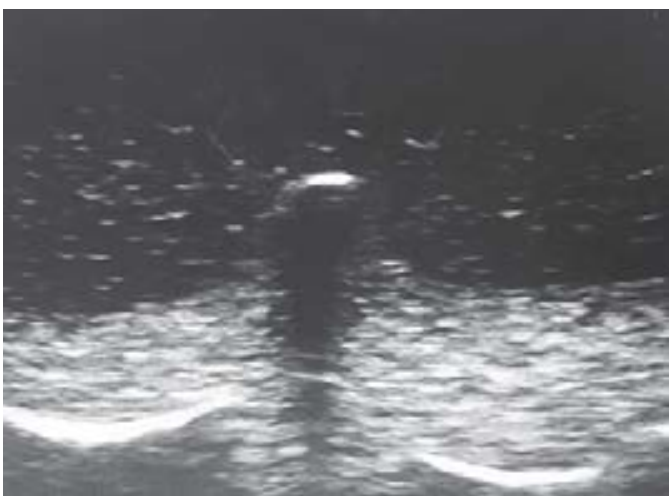

Figura 4. Imagen ultrasonográfica obtenida del simulador desarrollado: parasagital. la adquisición de conocimientos mediante el uso de estas técnicas, 111 contestaron que sí mejoraba; es decir, $75.5 \%$; 31 , que no (21\%), y cinco no respondieron (3.5\%).

\section{DISCUSIÓN}

El modelo diseñado permite abordar mediante técnicas de simulación el bloqueo neuroaxial de la región lumbar bajo control ultrasonográfico de bajo costo y alto impacto en los usuarios. Hace posible tener disponible un simulador apto para el entrenamiento médico en las técnicas descritas con alta satisfacción de los usuarios, como se ha expresado. Si bien el material es durable, las limitaciones que presenta el gel con el cual se confeccionó son las relacionadas a la exposición del mismo a altas temperaturas, ya que se desnaturaliza, y permite un número limitado de maniobras de punción. Si bien es durable, los reiterados procedimientos de punción deterioran la calidad de imagen obtenida cuando se superan los 150 a 200 usos. Dicho esto, consideramos que es una herramienta útil para el entrenamiento basado en simulación.

El entrenamiento basado en simulación es una técnica, no una tecnología, ${ }^{6}$ que consiste en sustituir la realidad por un escenario simulado en el que los estudiantes y profesionales pueden entrenar para adquirir habilidades - ya sean técnicas, psicomotrices, de comunicación o de trabajo en equipo-, que los preparará para la práctica clínica. La competencia profesional de los médicos se define como la práctica basada en el conocimiento médico, el razonamiento clíni$\mathrm{CO}$, el juicio crítico y las habilidades técnicas necesarias para actuar en beneficio de los pacientes y de la comunidad a la que pertenecen. ${ }^{7}$ Ello resulta sumamente relevante considerando el nivel de especialización que ha ido adquiriendo la práctica médica, lo que añade conocimientos y habilidades específicas que resultan necesarias para los profesionales de la salud. Por ello, en los últimos años, los organismos de acreditación universitaria han reconocido la importancia del entrenamiento basado en simulación y exigen la incorporación de esta técnica dentro de los programas de formación, tanto en el grado como en el postgrado. Las técnicas de simulación permiten entrenar a los profesionales con el objeto de crear un ambiente de seguridad para los pa- 
cientes mediante la práctica de procedimientos previamente ensayados en simuladores. En este sentido, el entrenamiento basado en simulación como una de las técnicas de educación médica permite no sólo que el estudiante adquiera habilidades técnicas, sino que pueda aprender de sus errores, reiterar maniobras y procedimientos, y anclar conocimientos teóricos mediante el "hacer"; además, hace posible su evaluación, la evaluación por pares y la autoevaluación. Este modelo de entrenamiento se encuentra basado y centrado en el estudiante y no en el docente, convirtiéndose este último en tutor. De este modo, se persigue que el estudiante sea artífice de su propio conocimiento y pueda percibir y percibirse como sujeto activo en el proceso enseñanza-aprendizaje. La utilización de simuladores como el diseñado, de complejidad baja, que permite entrenar maniobras específicas, acorta la curva de aprendizaje de las habilidades, y se ha demostrado que éstas son mejores que las curvas basadas en el entrenamiento clásico; ello obedece a que el simulador permite repetir el entrenamiento tantas veces como sea necesario, las habilidades adquiridas mediante la simulación son transferibles a la realidad inmediatamente. También, la introducción de las técnicas de entrenamiento basadas en simulación permite generar un doble mecanismo de seguridad: para los pacientes (al disminuir los errores médicos) y para los profesionales (que han podido enfrentar los escenarios de simulación antes de su práctica clínica). Estas circunstancias imprimen a las técnicas de entrenamiento basadas en simulación un perfil de rapidez y efectividad comparadas con las técnicas clásicas de educación médica. ${ }^{8}$

También debemos considerar que el acortamiento de la curva de aprendizaje redunda en beneficios, considerando la realidad actual de los profesionales de la salud, ya que éstos suelen disponer de menos tiempo para capacitarse. En los espacios de formación médica debe fomentarse la integración del saber y el hacer; cuando esto se logra, se genera un aprendizaje significativo. La capacidad de resolver situaciones complejas en el marco de la práctica profesional está dada por la suma de las destrezas, que deben encontrarse sostenidas y soportadas con el hacer. El uso de simuladores como el diseñado para la práctica de procedimientos invasivos ha demostrado gran utilidad en numerosos estudios, ${ }^{8}$ en particular, equiparando la habilidad y destreza en el procedimiento efectuado por expertos y por principiantes cuando los últimos han sido entrenados con estas técnicas. A partir del diseño y realización del modelo descrito, se ha podido establecer un nexo entre los contenidos teóricos propiamente dichos y la práctica clínica mediante la aplicación de estos conocimientos en un escenario simulado. De esta manera, se logra una herramienta útil, eficiente y segura que no presenta problemas éticos o legales a la hora de adquirir habilidades prácticas, lo que permite una adecuada transición desde los conocimientos teóricos hacia la práctica clínica. En el último tiempo, se está asistiendo al uso generalizado de estas técnicas en la formación de los médicos y profesionales de las ciencias de la salud, ya sea en el grado, el postgrado o la formación continua. Así, el entrenamiento médico basado en técnicas de simulación se ha convertido en una herramienta pedagógica fundamental para garantizar el aprendizaje de los médicos, mejorando la seguridad del paciente; "el uso de las simulaciones puede, por un lado, hacer más adecuada la formación de los profesionales, y a la vez, contribuir a minimizar el referido conflicto ético". ${ }^{5}$ Además, el entrenamiento basado en simulación permite que el error médico sea llevado hasta sus últimas consecuencias sin repercusiones reales; de este modo, el estudiante puede enfrentarse a diferentes situaciones de complejidad creciente en un ambiente seguro donde puede aprender de sus errores. Los errores son experiencias de aprendizaje $\mathrm{e}^{9}$ que deben ser capitalizadas tanto por los estudiantes como por los docentes.

Cabe pensar, por último, cuál es el rol que tienen los conocimientos anatómicos en relación con modelos de simulación y con la educación médica actual. En su conjunto, deben convertirse en herramientas que sumen la construcción del conocimiento, aportando datos útiles y habilidades que construyan competencias dentro de un proceso de aprendizaje significativo. Un modelo de educación médica centrado en el entrenamiento basado en simulación - que presente al profesional en formación o al estudiante un proceso secuencial que transite por la observación, la emulación y la repetición de técnicas en escenarios clínicos que sumerjan al estudiante en una realidad simulada que guarde el más fiel 
grado de realismo posible y la realización de técnicas más o menos complejas- garantiza una adecuada transición -ética, eficiente y segura- hacia la práctica clínica. El diseño de herramientas pedagógicas de la naturaleza que se presenta intenta sumar un componente más al inventario con el que se cuenta actualmente.

\section{CONCLUSIONES}

Se ha diseñado y desarrollado un simulador (fantoma) para la realización de bloqueos neuroaxiales guiados por ultrasonografía: de los primeros disponibles con estas características y construido en el país con materiales nacionales a un costo sensiblemente menor que el de los que se encuentran en la actualidad en el mercado. Este simulador se inscribe dentro de los disponibles para el entrenamiento médico basado en técnicas de simulación. Estas técnicas tienen como objeto que el profesional de la salud adquiera habilidades que le sean de utilidad en su práctica clínica. Hoy, el principal desafío que enfrentamos en el campo de la educación médica es generar investigaciones, diseños y desarrollos que tiendan a generar una forma más efectiva y eficiente de usar la simulación en el aprendizaje, de modo que se optimice el uso de recursos y se aporte a la formación profesional de calidad; esto redundará en la optimización de los procesos, la disminución de la tasa de errores médicos y la génesis de profesionales con altos niveles de formación y capacitación profesional. ${ }^{10}$

Para concluir, el uso de un simulador para la realización de bloqueos neuroaxiales guiados por ultrasonografía aplicando las técnicas de simulación para la educación médica, particularmente en regiones anatómicas o procedimientos complejos, es una estrategia dinámica y positiva para la formación profesional y por la que se adquieren habilidades que posibilitan prácticas análogas; éste es un campo rico para explorar y desarrollar mediante técnicas innovadoras y creativas - sin olvidar que imitan pero no sustituyen la realidad de la práctica clínica-4, ${ }^{41-14}$

\section{Agradecimientos}

A la primera Cátedra de Anatomía de la Facultad de Medicina de la Universidad de Buenos Aires.

\section{REFERENCIAS}

1. Matiz-Camacho H. La simulación clínica: nueva herramienta para enseñar medicina. Medicina (Bogotá). 2012; 34 (3): 242-246.

2. Vázquez-Mata G, Gullamet-Lloveras A. EI entrenamiento basado en simulación como innovación imprescindible en la formación médica. Educ Med. 2009; 12 (3): 149-155.

3. Hadzic A. Bloqueo de nervio periférico de Hadzic. 2. ${ }^{a}$ edición. Vol. 2. Amolca; 2015.

4. McDonald A, Murgatroyd H. Anestesia neuroaxial guiada por ultrasonido. World Federation of Societies of Anaesthesiologist, ATOTW, Tutorial 349 (21/03/2017).

5. Ziv A, Wolpe PR, Small SD, Glick S. Simulation-based medical education: an ethical imperative. Acad Med. 2003; 78 (8): 783-788.

6. Gaba DM. The future vision of simulation in health care. Qual Saf Health Care. 2004; 13 Suppl 1: i2-i10.

7. Pradell-Alentá H. El médico del futuro. Barcelona: Fundación Educación Médica; 2009.

8. Moya RP, Ruz AM, Parraguez LE, Carreño EV, Rodríguez CAM, Froes MP. Efectividad de la simulación en la educación médica desde la perspectiva de seguridad de pacientes. Rev Méd Chile. 2017; 145 (4): 514-526.

9. Ziv A, Berkenstadt H. La educación médica basada en simulaciones. Jano: Medicina y Humanidades. 2008; 1701: 42.

10. Dávila-Cervantes A. Simulación en educación médica. Inv Ed Med. 2014; 3 (10): 100-105.

11. Lizaraso-Caparó F. Simuladores para la enseñanza de la medicina o simulación de la enseñanza. Rev Horiz Med. 2012; 12 (1): 6-7.

12. Carrillo-Esper R, Díaz Ponce-Medrano JA, HuescaJiménez G, Ibarias-Enciso IA, Garnica-Escamilla MA, Cruz-Gutiérrez EO. Ultrasonografía del espacio epidural lumbar. Rev Mex Anest. 2017; 40 (2): 134137.

13. Okuda $Y$, Bryson EO, DeMaria $S$ Jr, Jacobson L, Quinones J, Shen B, et al. The utility of simulation in medical education: what is the evidence? Mt Sinai J Med. 2009; 76 (4): 330-343.

14. Ávila DR, Mahana TP, Rivera PC, Coll CP. Simulación clínica como método de formación de competencias en estudiantes de medicina. Rev Educ Cienc Salud. 2016; 13 (1): 11-14.
Correspondencia:

Santiago Maffia Bizzozero

Primera Cátedra de Anatomía, Facultad de Medicina (UBA).

Paraguay $2155,3^{\circ}$ piso (Sec. Uriburu), Ciudad Autónoma de Buenos Aires, Argentina.

Tel: +541159509500

(Int. 2114) / +541152853160

E-mail:smaffia@fmed.uba.ar 